\title{
L'orientation client ou comment faire travailler le consommateur
}

Jean-Claude De Crescenzo et Bernard Floris

\section{(2) OpenEdition}

1 Journals

Édition électronique

URL : http://journals.openedition.org/communicationorganisation/3234

DOI : 10.4000/communicationorganisation.3234

ISSN : $1775-3546$

Éditeur

Presses universitaires de Bordeaux

Édition imprimée

Date de publication : 1 juin 2005

Pagination : 19-30

ISSN : 1168-5549

\section{Référence électronique}

Jean-Claude De Crescenzo et Bernard Floris, "L'orientation client ou comment faire travailler le

consommateur », Communication et organisation [En ligne], 27 | 2005, mis en ligne le 07 juin 2012 , consulté le 19 avril 2019. URL : http://journals.openedition.org/communicationorganisation/3234 ; DOI : 10.4000/communicationorganisation.3234

Ce document a été généré automatiquement le 19 avril 2019

(c) Presses universitaires de Bordeaux 


\title{
L'orientation client ou comment faire travailler le consommateur
}

\author{
Jean-Claude De Crescenzo et Bernard Floris
}

1 Le client est roi, le client est au centre. Il est aujourd'hui personnalisé. C'est le dernier maître mot d'une pratique plus ancienne, celle de la fidélisation. Les entrepreneurs savent depuis longtemps qu'il revient beaucoup moins cher d'investir pour garder des clients, que d'investir pour reconquérir les parts de marché perdus. La fidélisation est devenue une préoccupation obsédante des services commerciaux du fait de la «volatilité » ou de la «versatilité » des clients, et du fait de la pugnacité de la concurrence.

2 Nous aborderons la question dite de «la fidélisation et de la personnalisation du client " d'un point de vue sociologique et communicationnel. Nous souhaitons rechercher le type de rapport social, relationnel et symbolique, exprimé sous forme gestionnaire et économique lorsque les entrepreneurs parlent de marketing relationnel, one to one ou expérientiel, évoquant par là les formes actuelles de la fidélisation, de récupération ou de conquête des clients par leur personnalisation présumée.

\section{L'orientation client comme régime de mobilisation de la force de travail}

3 Durant les Trente glorieuses, l'organisation fordienne correspondait à une production et un marché de masse où les besoins matériels à pourvoir furent un temps plus larges que les produits mis en marché.

4 L'écart se réduisant, les produits ont été considérablement diversifiés, leur cycle de vie s'est raccourci et l'amplitude des segments est devenue plus variable. La réactivité au marché et à la concurrence d'une part, et l'informatisation des processus de production d'autre part, ont considérablement ralenti le délai entre la conception et la mise en marché. Dans le même temps, l'industrialisation de la distribution a entraîné un écoulement beaucoup plus volumineux et rapide des marchandises. 
5 L'organisation fordienne ne correspondant plus aux transformations du marché de masse, les managers ont recherché de nouvelles formes d'organisation de l'entreprise et de relation avec le marché. En France, le management participatif a constitué la première étape historique d'une mutation radicale des politiques de management des entreprises, et le marchepied d'une politique de flexibilisation à tous niveaux, des appareils comme des hommes. Sans la participation des salariés à la définition du travail, cette politique de flexibilisation n'aurait pas été possible.

\section{Dictature et prévisibilité du comportement client}

6 Les discours managériaux sur la centralité du client et son importance capitale dans la vie et la survie de l'entreprise peinent à masquer le fait que, placé au cœur de l'entreprise, celui-ci devient plus facilement contrôlable. Il s'agit tout autant de contrôler le comportement d'achat et la forme de relation sous-tendue, que de créer, mesurer, prévoir ou anticiper les besoins dont il fera preuve.

7 L'irruption du client, puis la centralité de sa place dans le process de production et dans l'organisation du travail, n'ont été rendues possibles qu'à l'aide de règles, de pratiques, d'usages et aussi d'un imaginaire singulier et social. Les technologies qui interviennent dans la confrontation de l'entreprise aux besoins du client sont à la fois fonctionnelles, au sens où, issues de l'organisation du travail, elles servent principalement à modifier les processus de production et de mise en marché. Nous intégrons dans ces technologies le marketing direct, le CRM, le flux tendu, le système d'informations, la Qualité totale. Mais la technique ne peut être évaluée et analysée que si est également analysé l'imaginaire qui la construit. Nous intégrons dans ce paradigme l'ensemble des croyances, des significations, des symboles que nous retrouvons instrumentés dans les discours de communication, les politiques de gestion des ressources humaines, les codifications procédurales et prescriptives, les formes d'animation des équipes de travail, les politiques de management, et plus généralement toutes les formes de verbalisation tendant à construire dans l'entreprise une croyance collective. La Figure totémique du Client interdit toute pensée qui pourrait le desservir, et toute action limitant le service qu'il attend. Le totem ne se discute pas. Il se place en pré-requis ou postulat, à partir desquels une réflexion seconde, mineure puisque technique peut alors être instruite. Mais cette docilisation des consciences ne peut intervenir sans un accord préalable des salariés, accord au moins implicite, parfois contraint et forcé, mais le plus souvent volontaire.

\section{Le salarié assujetti par le client}

8 La centralité du client se présente donc comme un point de vue asservissant l'ensemble des processus organisationnels. Le client préside aux critères de qualité, de délai et de prix. Il prédétermine les fonctions mais aussi les relations entre les fonctions, entraînant des réactions en chaîne. Toute demande du client, codifiée ou non, a une répercussion immédiate sur l'ensemble du processus. Le client régule, le client contrôle, le client sanctionne. Ce qui hier était dévolu à la hiérarchie traditionnelle a été transféré au client (Linhart, 1994). Ainsi dans l'organisation se construit un dispositif implicite d'autorité nouvelle, non organisationnelle, qui va de la surveillance au contrôle et à la sanction en cas de non conformité à la commande. 
9 Il s'agit dans cette dernière période de ne plus créer d'écart entre le travailleur et le consommateur. Par sa place centrale dans l'entreprise, la présence du client devient capitale et se pose comme une figure tierce, à partir de laquelle, même absent, le client reste présent. Figure indiscutée puisque indiscutable qui a le parfait mérite de fabriquer le consensus organisationnel dont l'entreprise a besoin. Dans cet univers pacifié, qui pourrait mettre en doute, voire s'opposer à la demande du client, quand chacun de nous est aussi client à son tour ? La place du client est probablement la forme la plus douce de pacification des relations sociales, historiquement conflictuelles en France.

\section{Extension du domaine de la concurrence...}

10 Le passage de l'organisation fordienne à l'entreprise flexible orientée client traduit bien plus que la simple mise en adéquation de l'entreprise à un nouveau marché de masse " customizé ». En deçà de l'apparence économique et organisationnelle de ce processus, il nous semble apercevoir une transformation structurelle des rapports sociaux, dans leurs dimensions à la fois relationnelles et symboliques. Le passage d'un marketing transactionnel à un marketing relationnel traduit cette transformation en termes managériaux.

\section{Marketing relationnel versus marketing transactionnel ?}

11 Comment les évolutions du marché sont-elles enregistrées par les entreprises? Progressivement, la masse des produits diversifiés mis en vente a largement dépassé les capacités d'absorption du marché. Le marketing mix et les fameux "4P" étaient au centre de la forme dite transactionnelle. Ces derniers constituent une définition du marketing orienté vers le produit en fonction de segments de masse. La grande distribution a eu dans un premier temps une fonction de rationalisation et de contrôle de la transaction entre vendeur et acheteur, alors que la publicité stimulait les appétits des consommateurs.

Dans le même temps, l'industrialisation et la marchandisation des services développaient un marché plus vaste que celui des biens manufacturés. Dans les industries de services, la relation avec le client, et son intégration de fait dans la production du service est apparue comme une donnée décisive de création de valeur. Au-delà de la transaction se révélait l'importance de la relation, non seulement au moment de la consommation du service, mais dans sa perpétuation. Au même moment, poussées à la fois par la concurrence et par les exigences des consommateurs, les industries de biens découvraient l'importance du suivi de la relation commerciale dans les services après-vente.

Ces deux tendances conjointes pressaient les entreprises de s'adapter. C'est ce qu'a traduit la création d'un marketing relationnel (Kaufman, 1997 ; Hetzel, 2003). Le suivi et la pérennité de la relation commerciale, favorisés par les Tics, se sont avérés un avantage concurrentiel et un moyen de satisfaction des consommateurs, d'abord dans les industries de services, puis dans les industries de biens, devenant elles-mêmes en partie du service. La stratégie traditionnelle de marketing mix centrée sur le produit s'est avérée dès lors insuffisante. Le contact et l'interactivité avec le client devenaient une nécessité pour le garder ou le conquérir. L'adaptation plus fine aux besoins présumés des clients, le service 
après-vente et le maintien de la relation commerciale ont rapidement été suivis par l'idée d'une « personnalisation » du produit.

\section{L'extension de l'ère de contrôle de l'entreprise sur le marché et sur l'espace domestique}

Comment analyser ce passage présumé du «transactionnel» au «relationnel» sous l'angle sociologique et communicationnel ? On ne peut oublier que le marketing concept s'est aussi constitué dans la foulée des soubresauts sociétaux des années soixante-huit. Et il est probable que la réponse directe à un problème commercial était aussi une réponse indirecte à une problématique sociale plus générale. Cela d'autant plus que la première vague d'anticonsumérisme est apparue alors, et qu'elle était parallèle à une forte vague de critique du capitalisme et de la société de marché.

Aux risques d'une dévalorisation sociale de la consommation marchande, les entreprises ont répondu par la diffusion d'un mode de vie encore plus consommatiste, tout en récupérant les critiques sociales. Dans l'entreprise, les aspirations autogestionnaires étaient récupérées par le management participatif. Sur le marché, les aspirations à la démassification et à l'authenticité des marchandises (Boltanski, 1999) étaient récupérées par leur diversification et leur renouvellement plus rapide. Le marketing dit «transactionnel », correspondait à la rationalisation et au contrôle du rapport social entre les entreprises et le marché en fonction des produits. Après avoir marginalisé le petit commerce, la grande distribution a industrialisé le processus de vente et étendu le contrôle rationnel au commerce de détail. Dans les services de masse, le rapport était déjà intégré du fait de la juxtaposition entre production et distribution.

6 La connaissance et le suivi des consommateurs ont été plus lents dans le même temps où ces derniers acquéraient à la fois eux-mêmes une expérience de l'échange et une exigence plus élevée, en matière de prix, de qualité, et de service. Cette maturation s'accompagnait d'un désir accru de consommation, stimulé par la diversité des produits, l'augmentation des revenus, le développement du marketing direct, et la croissance de l'incitation publicitaire. Le consommateur est devenu plus exigeant dans ses choix parmi les produits et les marques, pour des raisons immédiatement fonctionnelles et pour des raisons sociales plus profondes. Il a accordé dans le même temps plus d'importance à la consommation dans ses modes de vie, voire dans ses raisons de vivre.

La puissance accrue des bases de données a permis d'établir des tactiques de continuité avec les clients, l'individualisation des messages publicitaires par des opérations promotionnelles ciblées, et l'apparente personnalisation des offres au sein de segments plus fins. Personnaliser consiste en fait à utiliser la corrélation entre les différents achats pour relancer « personnellement » ceux qui se ressemblent. Mais y a t-il eu pour autant la formation d'un lien plus relationnel?

Le sociologue ne peut pas prendre pour argent comptant l'affirmation d'un passage du transactionnel au relationnel, tout en supposant que l'orientation client traduit probablement un processus social plus vaste. Un chercheur en marketing nous indique les pistes d'une déconstruction de la doxa du marketing modernisé (Marion, 2001). Il doute en effet que le marketing relationnel soit un nouveau paradigme. Il pense qu'il n'est qu'une extension des méthodes antérieures incitée par la pression conjointe de la concurrence intensifiée et des nouvelles technologies. 
19 Pour Philippe Marion, c'est la base de données qui a une structure relationnelle avec une mémoire illimitée des achats d'innombrables clients et le croisement de fichiers produits, clients, transactions et historiques. On est loin de relations basées sur un échange mutuel des acteurs (comme dans le $B$ to $B$ ). L'individu consommateur reste passif vis-à-vis du pilotage de ses actes d'achat par le marketing. «C'est moins le consommateur qui a besoin de relations durables que le marketer qui cherche à adapter sa pratique aux nouvelles formes concurrentielles créées par la gestion individualisée des contacts qu'autorise le changement technologique » (Marion, 2001, p. 9)

Il apparaît ainsi que la figure du client est tout aussi fabriquée que ne l'était celle du consommateur, à la différence que la prestation marchande est plus singularisée. On saisit là toute l'ambiguïté du terme "relationnel», pensé rigoureusement comme extension du rapport marchand avec les acheteurs, et pouvant apparaître dans la foulée comme relation avec des personnes singulières. Mais Philippe Marion en reste trop à notre sens au rôle de la concurrence et des Tics. Si on envisage le processus marchand dans sa globalité, le moteur est plutôt celui de la nécessité d'étendre la sphère de contrôle des entreprises sur les comportements et les représentations des individus consommateurs afin de pérenniser le mode de vie consommatiste. Le professeur de marketing dit lui-même qu'il pourrait se « constituer des groupes de manière autonomes, voire des marchés de consommateurs à consommateurs, sans attendre que le marketer les "segmente "".

21 En effet, les «tactiques » des consommateurs ne cessent de contourner les «stratégies » des marketers (De Certeau) qui recherchent toujours plus de moyens de contrôler leurs comportements. Le passage présumé à un marketing « relationnel » exprime selon nous une extension de la maitrise de l'espace social général par les rapports marchands. Après la maîtrise rationalisée du rapport entre production et distribution, on est passé aujourd'hui à la maîtrise du processus entre production, distribution et consommation, via le contrôle de l'individu-client ${ }^{1}$. La croissance des sociétés de vente par correspondance, le développement accéléré du marketing direct, l'extension des cartes de fidélité dans la grande distribution, et la croissance du service après-vente dans tous les secteurs ont été la base de l'orientation dite « relationnelle » du marketing, avant même qu'il en prenne le nom.

\section{L'individualisation marchande à la rencontre de l'individuation anthropologique}

22 L'apparition du CRM et des formes dites one to one et expérientielles du marketing sont les techniques dites de personnalisation des produits et de la satisfaction des clients. L'analyse est ici complexe, tant il est difficile de discerner ce qui a été déterminant dans la rencontre de deux processus de nature différente, d'une part le processus d'individuation débuté depuis des siècles dans les sociétés occidentales, et accéléré après la seconde guerre mondiale; et d'autre part la pression d'individualisation des modes de vie, opérée par la marchandisation accélérée des rapports sociaux, et en particulier de l'espace familial et domestique. On peut assimiler cette rencontre à l'entrechoc d'une volonté d'émancipation du « sujet » et d'un assujettissement individualisé.

23 Avant que n'émerge la démarche d'adaptation des biens et des services à des «demandes» individualisées, il a fallu un long travail de pénétration de l'espace domestique en vue de son contrôle marchand par l'entreprise. On ne mentionnera ici que 
trois étapes récentes de ce mouvement de pénétration. La consommation de masse s'est généralisée après la seconde guerre mondiale. Dans un premier temps, c'est l'équipement des foyers en appareils ménagers électriques, en appareils audiovisuels (radio puis télévision, électrophones puis chaînes Hi fi) et en automobiles qui a accompagné la consommation des biens courants. L'appareillage industriel entrait ainsi dans l'espace privé et établissait un moyen d'observation et de contrôle de la consommation. Dans un second temps, la grande distribution industrialisée, se faisait le relais principal de l'acte d'achat des ménages, et étendait encore plus l'observation et le contrôle de la consommation privée. À chacun de ces deux temps, correspondait un renforcement de la consommation marchande dans les modes de vie. Dans un troisième temps, l'industrialisation des services a détruit des activités jusque-là non-marchandes, et elle a inversement étendu la sphère marchande des biens manufacturés aux services industriels et marchands. Les conditions étaient ainsi créées d'un passage de la «transaction » à la "relation », c'est-à-dire d'une extension de l'interpénétration de l'entreprise et du marché, et du contrôle du second par le premier.

Dans ces trois temps, le mode de vie consommatiste a conquis l'espace public et privé. Dans l'espace public, la multiplication des « Centres commerciaux » comme lieux d'achat, de loisirs et de détente hebdomadaire en est la plus spectaculaire expression. Dans l'espace privé, la multiplication des technologies d'usage privatif a renforcé l'individualisation des pratiques de consommation.

Le troisième temps correspond à la globalisation mondiale des marchés. A ce moment se sont enchevêtrées l'individuation anthropologique et l'individualisation marchande. L'aspiration historique à l'autonomie par rapport aux contraintes autoritaires des institutions et aux valeurs religieuses, morales ou politiques traditionnelles, a trouvé dans les pratiques marchandes familiales et privatives de consommation un de ses plus forts relais, cela d'autant plus que se marchandisaient des pratiques sociales autoproductives. Depuis cinquante ans, la publicité n'a cessé de vanter un individu consommateur hédoniste et libre de soi, en relayant les aspirations à la liberté, à l'amour, au confort, au plaisir, à la beauté, à la jeunesse, et en les accrochant systématiquement aux marchandises comme moyen d'y parvenir.

C'est ce processus d'individuation sociale et d'individualisation marchande, et non l'inverse, qui est à l'origine du fantastique développement des "Tics », lesquelles l'ont évidemment amplifié en lui imprimant leurs cadres de pratiques et de représentations. En vingt ans à peine ont explosé les chaînes de télévision, le lecteur de $\mathrm{Cd}$ puis de $\mathrm{Dvd}$, le walkman, le microordinateur personnel, les consoles de jeu, l'Internet, le téléphone portable, avec la variété d'usages et de services offerts par ces appareils. Leur première caractéristique est qu'ils favorisent systématiquement des pratiques individuelles. Cette individualisation est d'autant plus favorisée que ces appareils se renouvellent de plus en plus rapidement, captant ainsi encore plus l'intérêt et l'attention des consommateurs. L'obsolescence accrue des produits est ainsi à la fois matérielle (usure) et subjective (lassitude). Leur deuxième caractéristique est qu'ils permettent une connaissance des pratiques individuelles de consommation, et des réponses modularisées aux apparences de personnalisation en termes d'offre de services. On assiste à la fois à une fidélisation grandissante des clients à ces appareils, quelle que soit leur marque, et au-delà à une fidélisation globale des individus à la consommation de Tics individualisés.

Par ailleurs, les Tics ont opéré une mutation énorme dans la collecte directe et le traitement en temps réel des comportements d'achat et des caractéristiques personnelles 
des clients. D'immenses bases de données (data mining, data warehouse) travaillent en temps réel à croiser des milliards d'informations personnalisées et à les confronter aux « offres » de produits. La gestion personnalisée du client (CRM), le marketing de réseau, les questionnaires par mailing ou Internet, les relevés de cartes bleues ou de cartes de fidélité, les enquêtes par sondage et les centres d'appel sont des outils puissants pour suivre et contrôler le comportement des consommateurs. Cela a permis à toutes les industries de biens et de services d'établir un contact suivi avec leurs clients, de leurs offrir des services complémentaires à leurs achats, de leur proposer des promotions ou le renouvellement de leurs biens, de les informer régulièrement de leurs produits, et prélever en retour des informations personnelles.

\section{Le consommateur travailleur et créateur de valeur}

28 L'équipement des foyers en appareils industriels ménagers, audiovisuels et informatiques a inauguré un travail rationalisé et contrôlé du consommateur dans les cadres prévus ou prescrits par les entreprises ${ }^{2}$. Cette industrialisation de la vie privée a préparé sa personnalisation, laquelle a été particulièrement accélérée avec l'introduction des Tics. Cette rationalisation et ce contrôle matériels de l'espace domestique ont en même temps été accompagnés de la diffusion dominante d'un nouvel imaginaire marchand légitimant le mode de vie consommatiste. Ces deux technologies de pouvoir opérationnelles et symboliques sont complémentaires et inséparables, alors que la seconde tend à devenir de plus en plus déterminante.

\section{Du contrôle direct au contrôle à distance}

La rationalisation et le contrôle du travail dans les entreprises ont été fondés sur le commandement hiérarchique et l'obéissance des exécutants. Longtemps, la direction du personnel s'est établie à partir de relations directes entre chefs et subordonnés dans l'espace fermé de l'entreprise. Michel Foucault (1975) a assimilé ce mode de direction à un dispositif disciplinaire de contrainte et de formatage des corps, dont le "Panoptikon" carcéral est le symbole idéal. Un lent processus de desserrement de la contrainte hiérarchique dans toutes les institutions s'est opéré à partir des années soixante.

C'est ce qui a conduit Gilles Deleuze (1990) à faire l'hypothèse du passage à un autre régime de pouvoir fondé sur un nouveau dispositif de contrôle à distance dans des milieux ouverts. Il a identifié au passage le marketing, "nouvelle race des maîtres", comme l'instrument du nouveau contrôle social. Luc Boltanski a fait pour sa part l'hypothèse de la formation d'un "nouvel esprit du capitalisme " où les organisations ouvertes, en réseau et par projets sont le nouveau mode de contrôle. Si le chef tend à disparaître, c'est l'individu lui-même qui devient son propre chef en fonction des contraintes prédéterminées de son autonomie.

31 La démarche du marketing a été à l'origine d'un apprentissage managérial du contrôle à distance des activités. Acheter et consommer ne se fait pas sous contrainte. Tout l'art de la vente consiste à «faire penser et à faire faire à des publics cibles ce qu'on veut qu'ils pensent et ce qu'on veut qu'ils fassent» (Schwebig, 1986, p. 81) sans pouvoir leur ordonner de tâches prescrites, ni être « derrière leur dos " pour les surveiller. Cet énoncé est bien plus important qu'il n'y paraît dans sa simplicité. Il dit à la fois que le «faire penser" est une condition du "faire faire», et il exprime l'intention d'un "agir 
instrumental et stratégique» (Habermas, 1988) de la part des émetteurs de la communication vers leurs récepteurs.

C'est parce qu'il a créé par nécessité des démarches de contrôle des comportements et des représentations des consommateurs que le marketing s'est révélé être un régime de pouvoir plus efficace que la discipline et mieux adapté à des individus autonomes dans des milieux ouverts et fluctuants. Ainsi a t-il pu faire advenir concrètement le mythe de la « personnalisation » du client.

\section{CRM, centres d'appel et marketing direct : le contrôle du client à distance}

Les démarches opérationnelles les plus récemment apparues expriment la capacité du marketing à rationaliser le procès de consommation et à régler à distance l'autocontrôle des clients.

Le marketing direct a depuis longtemps instauré un contrôle des relations marchandes. A partir des ventes par correspondance et du mailing, il s'est développé en marketing de réseau (par téléphone puis Internet). Depuis moins d'une dizaine d'années, les centres d'appels se sont rapidement multipliés. Avec prêt de 200000 téléopérateurs, ils abreuvent quotidiennement les foyers de propositions de produits divers, et de promotions sur toutes les marques à des prospects. Ils se chargent aussi du suivi de la relation avec les clients.

Le Customer relationship management, ou gestion personnalisée des clients, est une des dernières créations du marketing direct. À partir de bases de données très sophistiquées sur les clients, il établit des stratégies de suivi personnalisé de millions de clients par des informations, des relances, des promotions, des concours en recherchant la pérennisation du contact en fonction de données personnelles. Si chaque client peut avoir la sensation concrète d'une personnalisation, celle-ci est en totale contradiction avec la rentabilité des produits. Les segments sont devenus plus souples, les cycles de vie des produits ont été réduits, et leur innovation accélérée, mais la standardisation n'a pas pour autant disparu. Ce qui apparaît comme personnalisé à tel client, correspond en réalité à une modularisation d'options prédéterminées pour un produit standard dans un même segment.

Si le contrôle opérationnel est motivé par l'efficacité commerciale aux yeux de ses promoteurs, il n'en exprime pas moins l'extension d'un contrôle social sur l'ensemble de la société. Avec les instruments traditionnels d'études de marché et d'encadrement du client, le marketing direct, les centres d'appel et le CRM ont considérablement étendu la puissance de contrôle du marché et le pouvoir de subsumption de toutes les relations sociales par les entreprises. À la différence de la contrainte directe sur les corps et les esprits enfermés dans les limites physiques de l'entreprise, le contrôle à distance du client ne peut se faire qu'avec sa collaboration consentante, voire enchantée. Celle-ci est d'abord conditionnée par l'emprise et l'envahissement d'un mode de vie auquel il est difficile d'échapper, et qui a toutes les apparences de la normalité indiscutable, sinon d'une véritable nature. 


\section{L'emprise d'une nouvelle signification imaginaire sociale} société par le biais des sciences, des techniques et de la production industrielle. Conjuguée à l'emprise de l'univers dominant de la consommation, c'est à notre sens une nouvelle signification imaginaire sociale qui s'est introduite dans les esprits depuis une trentaine d'années avec l'avènement de la figure du client. Ce dernier réunit l'acheteur, le consommateur et l'individu autoréalisateur de soi. Il est l'incarnation et l'institution de la croyance dans l'hédonisme sans fin et le désir illimité du nouveau grâce à la consommation marchande. En même temps, il permet de légitimer et d'enchanter l'énormité de l'appareil de rationalisation et de contrôle social des individus mis en place par les entreprises, via le marketing. Il est la base du processus d'intégration totale entre les entreprises et le marché, entre la production, la distribution et la consommation. La personnalisation du client est l'ultime expression de cet imaginaire, en même temps qu'elle exprime l'extension de la domination et du contrôle social par la market globalization.

\section{BIBLIOGRAPHIE}

Boltanski L., Chapello E.., Le nouvel esprit du capitalisme, Gallimard, 1999

Castoriadis C., L'institution imaginaire de la société, Seuil, 1975

De Certeau M., L'invention du quotidien ; arts de faire, Gallimard, 1990

Deleuze G., « Post-scriptum sur les sociétés de contrôle », in Pourparlers, Minuit, 1990 
Durand J-P, Hatzfeld N., La chaîne et le réseau, Pages deux, 2003

Foucault M., Surveiller et punir, Seuil, 19

Giedion S., La mécanisation au pouvoir, Denoël, 1980

Habermas J., Théorie de l'agir communicationnel, Seuil, 1988

Kaufman J-P, Le marketing de l'ego, Dunod, 2005

Linhart D., La modernisation des entreprises, La Découverte, 1994.

Marion G., « Le marketing relationnel existe t-il ? », Décisions Marketing, º 22, Avril 2001

Schwebig P., Les communications de l'entreprise, Mac Graw - Hill, 1986.

\section{NOTES}

1. Nous nous plaçons ici d'un point de vue social global. Au point de vue des entreprises et des marketers, ces outils sont vécus comme les réponses à une difficulté accrue à maîtriser un marché de plus en plus concurrentiel, et des consommateurs de plus en plus expérimentés, exigeants et volatiles. Mais ceci est valable du point de vue économique de chaque entreprise séparée. Au niveau économique global, la consommation se porte bien et les profits augmentent. $\mathrm{Au}$ niveau de la formation sociale, le rapport marchand étend de plus en plus son emprise sur la société et sur les individus. La «marchandisation du monde » n'exprime donc pas que l'extension de la forme marchandise à tous les objets, relations, et mêmes «expériences vécues » (Rifkin, 2000), elle exprime aussi l'extension de l'emprise sociale du mode de domination capitaliste sur toutes les sphères de la vie collective et individuelle.

2. Ces cadres prescrits correspondent à ce que Michel de Certeau (1990) appelle les « stratégies » des entreprises, pour exprimer leur position de domination par rapport aux « usagers (les consommateurs). Nous ne pouvons ici traiter des "tactiques" que ceux-ci emploient pour s'approprier ou détourner les usages prévus, mais qui ne peuvent se faire que dans les limites imposées par les stratégies des entreprises.

\section{RÉSUMÉS}

L'orientation client exprime les transformations organisationnelles des entreprises et leur nouveau rapport au marché. Le renouvellement d'un marketing transactionnel à un marketing relationnel exprime cette nouvelle voie de la fidélisation des clients via leur personnalisation affirmée. Cet article propose d'interroger ces transformations sous l'angle sociologique et communicationnel. Notre hypothèse est qu'elles sont l'expression d'une intégration de l'entreprise et du marché dans un système unifié et l'avènement d'un travail rationalisé et contrôlé des individus sous la figure du client.

The customer orientation expresses the organisational transformations of the companies and their new report at the market. Renewal of a transactional marketing to a relational marketing means this newway of the development of consumer loyalty of the customers with their supposed personalization. This article proposes to question these transformations under the 
sociological and communication point of view. Our assumption is that they are the expression of an integration of the company and market in an unified system and the advent of a work rationalized and controlled individuals under the figure of the customer.

\section{AUTEURS}

\section{JEAN-CLAUDE DE CRESCENZO}

Ex conseiller en communication organisationnelle, Jean-Claude De Crescenzo est maître de conférences à l'Université d'Aix-Marseille. Il travaille sur les transformations du management et sur la formation de nouveaux réseaux informels de travail dans l'entreprise flexible et globalisée. Mail : irco@wanadoo.fr

\section{BERNARD FLORIS}

Bernard Floris est maître de conférences Hdr à l'Université Stendhal Grenoble 3 et dirige le Centre d'études des dispositifs et processus de communication et d'information (Ced\&pic, JE 2315). Après avoir étudié la fonction des communications dans le néomanagement, il travaille actuellement sur l'intégration de l'entreprise et du marché à travers l'orientation client et la démarche généralisée du marketing. Mail :

roflisse@free.fr 